\title{
Unveiling ferrimagnetic ground state, anomalous behavior of the exchange-bias field around spin reorientation, and magnetoelectric coupling in $\mathrm{YbCr}_{1-x} \mathrm{Fe}_{x} \mathrm{O}_{3}(0.1 \leqslant x \leqslant 0.6)$
}

\author{
Biswajit Dalal, ${ }^{1, *}$ Babusona Sarkar, ${ }^{1,2}$ S. Rayaprol $\odot,{ }^{3}$ Moumita Das, ${ }^{4}$ V. Siruguri $\odot,{ }^{3}$ \\ Prabhat Mandal, ${ }^{4}$ and Subodh Kumar De (1) ${ }^{1, \dagger}$ \\ ${ }^{1}$ School of Materials Sciences, Indian Association for the Cultivation of Science, Jadavpur, Kolkata 700 032, India \\ ${ }^{2}$ Department of Physics, Sri Ramkrishna Sarada Vidya Mahapitha, Kamarpukur, Hooghly, West Bengal 712 612, India \\ ${ }^{3}$ UGC-DAE Consortium for Scientific Research, Mumbai Centre, Bhabha Atomic Research Centre, Mumbai 400 085, India \\ ${ }^{4}$ Saha Institute of Nuclear Physics, HBNI, 1/AF Bidhannagar, Kolkata 700 064, India
}

(Received 1 October 2019; revised manuscript received 3 February 2020; accepted 23 March 2020; published 13 April 2020)

\begin{abstract}
We present a comprehensive experimental study of the magnetic structure, magnetic and dielectric properties of the rare-earth orthochromite-orthoferrite solid-solution series $\mathrm{YbCr}_{1-x} \mathrm{Fe}_{x} \mathrm{O}_{3}(0.1 \leqslant x \leqslant 0.6)$. Roomtemperature synchrotron $\mathrm{x}$-ray diffraction analysis reveals the absence of any superlattice reflections, which excludes the formation of a $B$-site-ordered double-perovskite-like phase and establishes the complete solid solubility of $\mathrm{Fe}$ at the $\mathrm{Cr}$ site within the framework of orthorhombic Pbnm structure. We demonstrate that canted antiferromagnetic ground state of $\mathrm{YbCrO}_{3}$ is converted to a ferrimagnetic with $\mathrm{Fe}$ doping, in addition to an increase in the magnetic ordering temperature. An unusual, second magnetic transition (first-order in nature) appears for $x \geqslant 0.3$ samples below the ferrimagnetic transition temperature (e.g., at $70 \mathrm{~K}$ for $x=0.4$ ), which is identified as the spin reorientation of transition metal ions from the neutron powder diffraction measurements, and primarily, driven by the $f$ - $d$ exchange interaction. A clear evidence of the anomalous behavior of coercivity and exchange bias field is found around the spin reorientation temperature, which is characterized by a significant change in the magnetocrystalline anisotropy due to spin reorientation of transition metal ions. Temperature-dependent dielectric data exhibit the magnetoelectric coupling as well as a ferroelectric relaxor-like state at the onset of ferrimagnetic ordering. Here, we reveal the anomalous behavior of the exchange bias field and significant magnetoelectric coupling around the spin reorientation and ferrimagnetic transitions, respectively, in $\mathrm{YbCr}_{1-x} \mathrm{Fe}_{x} \mathrm{O}_{3}$.
\end{abstract}

DOI: 10.1103/PhysRevB.101.144418

\section{INTRODUCTION}

Recently, searching for materials with multiple ferroic orders has remained the thrust field of research for both theoretical and experimental material physicists, as the coupling between them allows one to manipulate one particular ferroic property via another, and explore some alluring physical properties, which could be used for the technological development of next-generation state-of-the-art multifunctional devices. Since the discovery of spin-induced ferroelectricity in centrosymmetric orthorhombic (Pbnm) $\mathrm{TbMnO}_{3}$ by Kimura et al. [1], single phase multiferroics (MFs), which simultaneously exhibit both magnetic and ferroelectric orders have attracted considerable attention to design and fabricate low-power spintronics and data storage devices using the spectacular cross coupling effects of the spin and charge degrees of freedom [2]. In general, this cross coupling is strong in the type-II MFs where the ferroelectricity appears due to particular kind of spin structure of the materials, as classified by Khomskii $[3,4]$. Owing to the mutually exclusive nature of magnetism and

\footnotetext{
*Corresponding author: b.dalal.iitd@gmail.com
}

†Corresponding author: msskd@iacs.res.in ferroelectricity, several mechanisms have been proposed so far to understand the origin of multiferrocity in type-II MFs, such as, breaking of the inversion symmetry through asymmetric Dzyaloshinskii-Moriya (DM) interaction, the strong spin-dependent $p$ - $d$ hybridization, the exchange striction, and the single-ion magnetic anisotropy [5-13]. However, this cross coupling of spin and charge can also lead to another aspect of the MFs i.e., the magnetoelectric coupling effect in the insulating materials apart from the proper ferroelectric polarization, which could facilitate the pathway to engineer memory devices based on this effect and enlighten our knowledge to model the cutting-edge multiferroic materials [14,15].

But, the main disadvantage of the type-II rare-earth manganite MFs is that the spin-driven ferroelectricity occurs at cryogenic temperatures (e.g., $\sim 27 \mathrm{~K}$ for $\mathrm{TbMnO}_{3}$ ) $[1,6,16,17]$. In this regard, rare-earth orthochromites of chemical formula $R \mathrm{CrO}_{3}$ (where $R=$ rare-earth element) have especially possessed a unique class of type-II MF materials due to their higher onset temperatures (133 to $197 \mathrm{~K})$ of spin-driven ferroelectricity compared to that of the rare-earth manganites [18,19], and since have been under investigation for quite some time as the complex interaction of the two magnetic sublattices of $R^{3+}$ and $\mathrm{Cr}^{3+}$ ions also gives rise to striking magnetic phenomena, such as, temperature induced 
magnetization reversal (MR), spin reorientation, zero-fieldcooled (ZFC) and field-cooled (FC) exchange bias, magnetoelastic coupling, and magnetocaloric effect [18-29]. These orthochromites mainly crystallize in centrosymmetric orthorhombic structure with space group either Pbnm or Pnma, and show weak ferromagnetism due to the canted arrangement of $\mathrm{Cr}^{3+}$ spins in their antiferromagnetic structure; while, the major magnetic contribution comes from $\mathrm{Cr}^{3+}-\mathrm{O}-\mathrm{Cr}^{3+}$ antiferromagnetic superexchange interaction.

In an earlier study [18], it was reported that the type-II multiferroic behavior in rare-earth orthochromites is prompted by the interaction between magnetic (only) rare-earth and weak ferromagnetic $\mathrm{Cr}^{3+}$ ions following the breaking of symmetry by the effect of poling. However, the emergence of multiferroicity in $\mathrm{LuCrO}_{3}$ (where rare-earth $\mathrm{Lu}^{3+}$ is diamagnetic in nature due to completely filled $f$ orbitals) has totally surpassed the previous perception, and highlighted that the magnetic nature of rare-earth ion is not mandatory to observe the multiferroic-like features [19]. Excluding the possibility of symmetry breaking below the transition metal ordering temperature in rare-earth orthochromites and orthoferrites (Pbnm), the authors of Ref. [30] considered the following assumptions: (i) pinning of the domain wall motion, (ii) magnetostructural distortion for the off-centering of $\mathrm{Cr}^{3+}$ ion, and (iii) mixed valency of $\mathrm{Cr}^{3+}$ ion, to explain the observed multiferroicity in $\mathrm{LuCrO}_{3}$. However, there is no report on the type-II multiferroicity for the previous member of this rareearth orthochromite series, i.e., $\mathrm{YbCrO}_{3}$, though $\mathrm{Yb}^{3+}$ ion has one unpaired $f$ electron that takes part in the magnetism. Instead, the presence of a high-temperature ferroelectric phase ( $\sim 439 \mathrm{~K}$ ) has been demonstrated from the temperature dependent dielectric measurements, which is well above the canted antiferromagnetic ordering of $\mathrm{YbCrO}_{3}\left(T_{N} \sim 120 \mathrm{~K}\right)$ [31]. Such a high-temperature ferroelectric phase in $\mathrm{YbCrO}_{3}$ is quite debatable and its origin has not been fully understood as of now.

Thus, the complex dielectric behavior along with the temperature induced MR phenomenon in both ZFC and FC mode, and ZFC exchange bias effect [27] makes using this particular material $\left(\mathrm{YbCrO}_{3}\right)$ fascinating to carry out the fundamental research. Moreover, the emergence of various extraordinary phenomena, such as, spin reorientation, metamagnetic transition, multiferroicity, and reversed exchange bias effect, in the complete solid-solution between rare-earth orthochromites, orthoferrites, and cobaltites [32-45] stimulated us to revisit the solid-solution of rare-earth orthochromites and orthoferrites, and explore some intriguing physical characteristics in more detail. In this context, we choose to investigate $\mathrm{YbCr}_{1-x} \mathrm{Fe}_{x} \mathrm{O}_{3}$, as the parent compound $\mathrm{YbCrO}_{3}$ exhibits many interesting magnetic properties; whereas, Fe doping might provide the suitable platform to induce complex magnetic ordering, and type-II multiferroicity (and/or, magnetoelectric coupling) through local charge ordering and/or, magnetic frustration [46].

In this paper, we report a detailed study of the roomtemperature synchrotron x-ray powder diffraction, temperature dependent neutron powder diffraction, and temperature and magnetic field dependent magnetic and dielectric properties of the solid-solution series $\mathrm{YbCr}_{1-x} \mathrm{Fe}_{x} \mathrm{O}_{3}(0.1 \leqslant x \leqslant$ 0.6). No miscibility gap is observed throughout the com- positional range from the structural refinement of roomtemperature synchrotron x-ray data, whereas lattice parameters almost follow linear behavior, mimicking the validity of Vegard's law. A clear transformation of the magnetic ground state with $\mathrm{Fe}$ doping is established from the analysis of inverse susceptibility data above magnetic ordering temperature. More importantly, using a combination of temperature dependent neutron powder diffraction and magnetization study, we show that transition metal spins for $x \geqslant 0.3$ samples reorient at a certain temperature, which is far below their onset of ferrimagnetic ordering. From the anomalous behavior of coercive field and exchange bias field, concomitant with the spin reorientation transition, we propose that the spin reorientation of transition metal ions significantly influences the magnetocrystalline anisotropy of the sample. In addition, we show that a possible magnetoelectric coupling, accompanying the ferroelectric relaxorlike state, exists around the onset of ferrimagnetic ordering for $x=0.3$ and 0.4 samples.

\section{EXPERIMENTAL DETAILS}

High quality single-phase polycrystalline samples of $\mathrm{YbCr}_{1-x} \mathrm{Fe}_{x} \mathrm{O}_{3}$ (where $0 \leqslant x \leqslant 1$ ) were synthesized by standard solid-state reaction of $\mathrm{Yb}_{2} \mathrm{O}_{3}, \mathrm{Cr}_{2} \mathrm{O}_{3}$ and $\mathrm{Fe}_{2} \mathrm{O}_{3}$ in air. Stoichiometric quantities of these starting raw binary oxides were mixed in an agate mortar, ground together for $1 \mathrm{~h}$ and calcined at $600{ }^{\circ} \mathrm{C}$ for $24 \mathrm{~h}$ in air. After furnace cooled, the resulting powder was then reground thoroughly for better homogeneity and pressed into a cylindrical pellet through $10 \mathrm{MPa}$ pressure using a uniaxial hydraulic press. The pellets were then sintered twice in air at 1150 and $1250{ }^{\circ} \mathrm{C}$, respectively, for $24 \mathrm{~h}$ with intermediate grinding for better crystalline phases. However, owing to large amount of powder samples required for neutron diffraction experiments, the powders were not pressed into pellets in order to obtain better oxygenated samples and sintered twice more at $1250{ }^{\circ} \mathrm{C}$ for $24 \mathrm{~h}$. The preliminary phase purity of all samples was checked by laboratory-based $\mathrm{x}$-ray diffraction $(\mathrm{Cu} K \alpha$ radiation, $\lambda=$ $1.5418 \AA$ A) measurements using $\mathrm{x}$-ray powder diffractometer (X-pert Pro, PANalytical) in the range $20^{\circ}-90^{\circ}$.

Room-temperature high-resolution $\left(\Delta E / E=2 \times 10^{-4}\right)$ synchrotron $\mathrm{x}$-ray powder diffraction measurements along with the standard NIST Si sample were performed on P02.1 High-Resolution Powder Diffraction beamline at the Petra III, DESY, Hamburg, Germany with a wavelength of $0.20737 \AA(\sim 60 \mathrm{keV})$ using a fast area two-dimensional (2D) Perkin Elmer (XRD1621) detector. The 2D synchrotron Xray diffraction images were calibrated and processed through FIT2D program [47]. Later, the 1D synchrotron diffraction data were interpreted through Rietveld refinement method [48] with the help of commercially available materials analysis using diffraction (MAUD) software [49]. The bond valence sum (BVS) values were calculated using the software SPUDS [50]. The magnetization measurements on powder samples were carried out using a Quantum Design MPMS XL Evercool superconducting quantum interference device magnetometer, heating from 2 to $300 \mathrm{~K}$ at 100 Oe magnetic field in both ZFC and FC protocols, and isothermal magnetization loops were collected within $\pm 50 \mathrm{kOe}$. To circumvent any contribution from the stray magnetic field of superconducting magnet, 

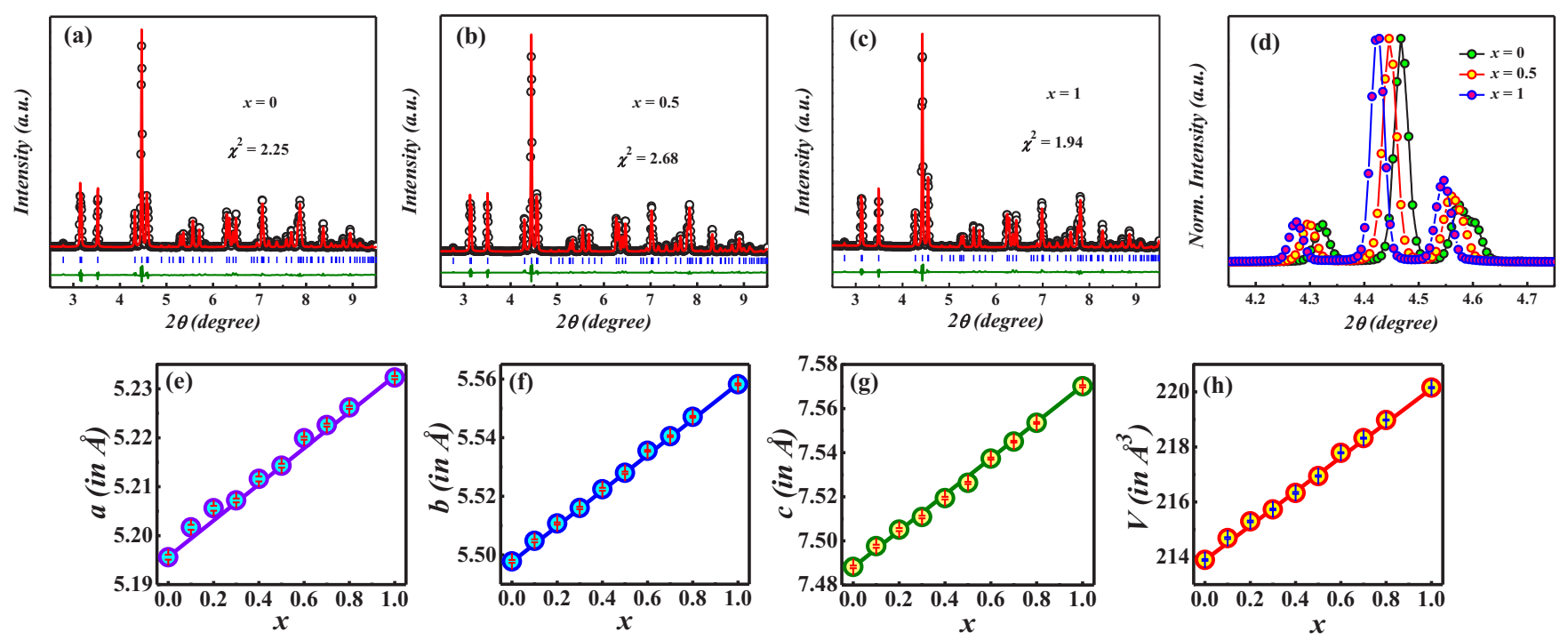

FIG. 1. Rietveld refinement of the room-temperature high-resolution synchrotron $\mathrm{x}$-ray powder diffraction patterns for (a) $x=0$, (b) $x=$ 0.5 , and (c) $x=1$ using orthorhombic (space group Pbnm) phase. (d) Shows the combined plot of diffraction patterns in (a)-(c) within $2 \theta=4.15^{\circ}-4.75^{\circ}$. (e)-(h) Compositional dependence of the structural parameters in $\mathrm{YbCr}_{1-x} \mathrm{Fe}_{x} \mathrm{O}_{3}$ (where $0 \leqslant x \leqslant 1$ ) series. The solid straight lines in (e)-(h) are guide to the eye.

the sample space of magnetometer was degaussed at each time before measurement, and then centering was done in presence of 100 Oe magnetic field at room temperature. Neutron powder diffraction (NPD) measurements were performed using polycrystalline samples, on a position sensitive detector based focusing crystal diffractometer PD-3 at Dhruva Reactor, Trombay [51] using neutrons of wavelength $1.48 \AA$. For temperature dependent measurements, a closed cycle refrigerator (CCR, M/s A S Scientific UK) was used. Samples, packed in vanadium cans, were attached at the end of the variable temperature insert (VTI) of the CCR. The dielectric constant and loss measurements were performed as a function of temperature $(T)$ down to $3 \mathrm{~K}$ in a VTI of a cryogen free measurement system (Cryogenic Ltd., UK) using an ultraprecession capacitance bridge AH 2700A (Andeen-Hagerling, Inc.). The temperature dependent thermal expansion measurement in zero field and in presence of an applied field (parallel to the sample's length for longitudinal magnetostriction) was performed on polycrystalline sample by a capacitive method using a miniature tilted-plate dilatometer.

\section{RESULTS AND DISCUSSION}

\section{A. Crystal structure}

Precise analysis of the powder diffraction data throughout the compositional range reveals that all samples crystallize in orthorhombic structure with space group Pbnm (No. 62). The Rietveld fitted diffraction data along with the difference spectra (between the observed and fitted patterns) and Bragg reflections for $x=0,0.5$, and 1 are shown in Figs. 1(a)-1(c), respectively. The values of the profile fitting parameter $\left(\chi^{2}\right)$ around $\sim 2$ represent the well-fitting of the diffraction data through refinement. The absence of any extra Bragg peaks in the diffraction patterns further ratifies the single-phase nature of the samples throughout the compositional range and rules out the evolution of any lower symmetric and/or, double perovskitelike structures with doping, i.e., no miscibility gap is observed in this solid-solution series. It is seen that the diffraction peaks are shifted towards lower angles with the incorporation of larger $\mathrm{Fe}^{3+}$ ion $(0.645 \AA)$ at the site of smaller $\mathrm{Cr}^{3+}$ ion $(0.615 \AA)$ in $\mathrm{YbCrO}_{3}$ [see Fig. 1(d)], indicating the enhanced unit cell volume for the doped samples.

In order to check the validity of Vegard's law throughout the compositional range, we have also determined the lattice parameters and unit cell volume for other compositions in this series from Rietveld fitting (Figs. S1 and S2 in the Supplemental Material (SM) [52]). The results of the crystal structure refinements for all samples in this series are summarized in Table S1 in the SM [52]. The variation of lattice parameters $(a, b$, and $c)$ and unit cell volume $(V)$ with compositions are depicted in Figs. 1(e)-1(h), which reveals the continuous increase in the corresponding value of the parameters with doping. A linear relationship of the lattice parameters and unit cell volume is found between the two end members [i.e., $\mathrm{YbCrO}_{3}(x=0)$ and $\mathrm{YbFeO}_{3}(x=1)$ ], justifying that Vegard's law holds true for this compositional series. Note that no change in the oxidation state is expected for $\mathrm{Cr}$ and $\mathrm{Fe}$ to maintain the charge neutrality in the doped samples, as both atoms carry equal positive charge. Despite this fact, to further confirm the oxidation states of $\mathrm{Cr}$ and $\mathrm{Fe}$, we have estimated the BVS values for $\mathrm{Cr}$ and $\mathrm{Fe}$ from the results of structural refinement. The best agreement between BVS values (Table $\mathrm{S} 1$ in the SM [52]) and different oxidation states suggests that $\mathrm{Cr}$ and $\mathrm{Fe}$ are present as $\mathrm{Cr}^{3+}$ and $\mathrm{Fe}^{3+}$ in the samples, respectively.

\section{B. Temperature dependence of magnetization}

The temperature $(T)$ dependent dc magnetization $(M)$ for $x=0-0.6$ [Figs. 2(a)-2(g)] in both FC and ZFC protocols is measured under an applied magnetic field $(H)$ of $100 \mathrm{Oe}$, where FCC and FCH curves represent the FC $M(T)$ data 

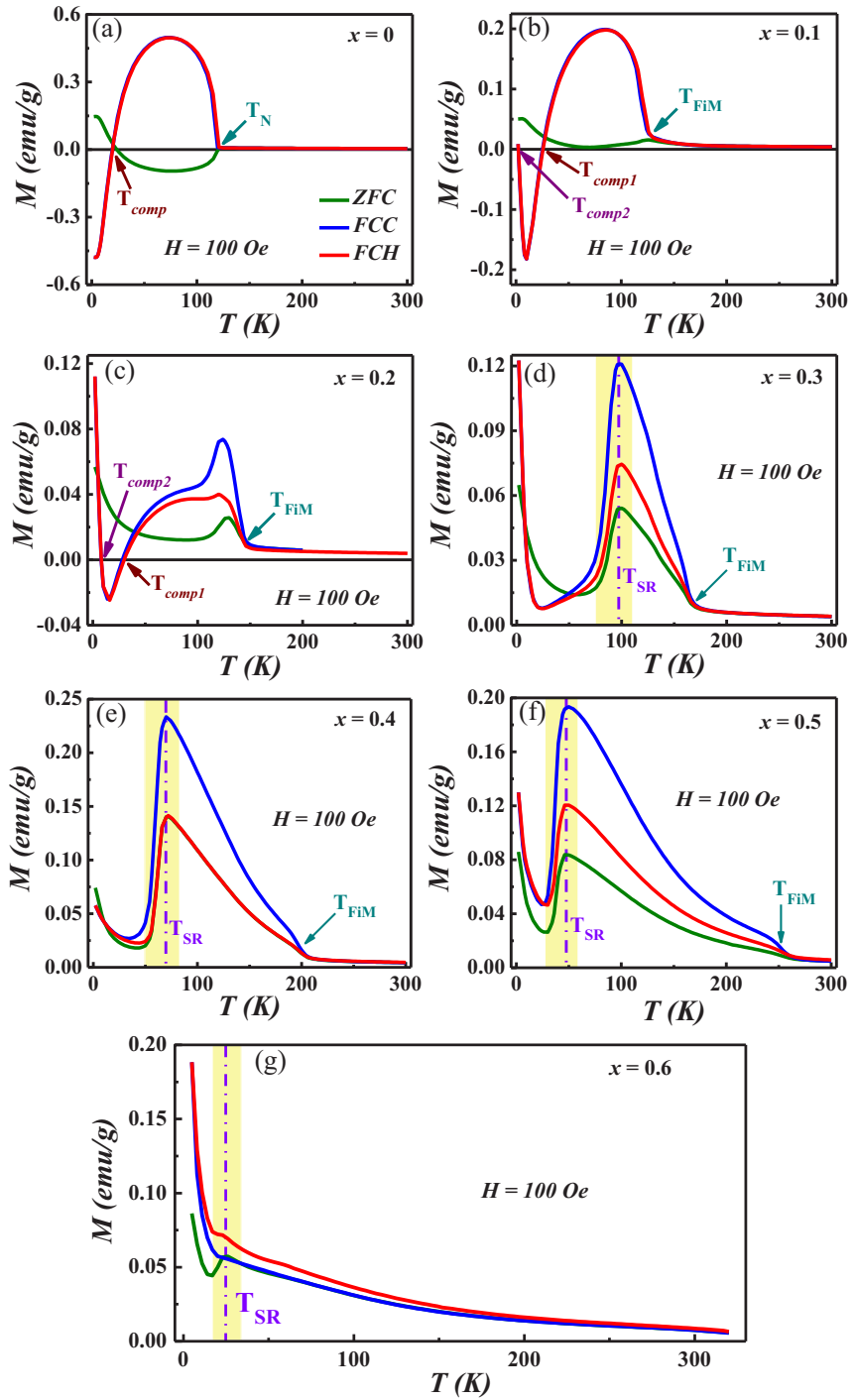

FIG. 2. Temperature (T) dependence of the ZFC, FCC, and FCH magnetization (M) of (a) $x=0$, (b) $x=0.1$, (c) $x=0.2$, (d) $x=0.3$, (e) $x=0.4$, (f) $x=0.5$, and (g) $x=0.6$ in a measuring magnetic field of $H=100 \mathrm{Oe}$. The dash-dotted lines in (d)-(g) represent the spin-reorientation temperature $\left(T_{S R}\right)$ of $\mathrm{Cr}^{3+} / \mathrm{Fe}^{3+}$ ions.

collected during cooling and heating processes, respectively. A sharp increase in the FCC magnetization value near $120 \mathrm{~K}$ reveals the onset of magnetic ordering for pure $\mathrm{YbCrO}_{3}$ i.e., $x=0$ [Fig. 2(a)], which is related to the antiferromagnetic (AFM) ordering $\left(T_{N}\right)$ of $\mathrm{Cr}^{3+}$ moment [27]. Below $T_{N}, x=0$ sample shows a broad maximum in FCC $M(T)$ at $75 \mathrm{~K}$, and the $M$ reaches the zero value at $T_{\text {comp }}=20 \mathrm{~K}$, showing negative values (magnetization reversal) upon further cooling. In addition, a small plateau $(<10 \mathrm{~K})$ is noticed in FCC $M(T)$ owing to the AFM ordering of rare-earth $\mathrm{Yb}^{3+}$ moments $\left(T_{N} Y b\right)$. Both FCC and FCH magnetization curves show similar kind of $T$-dependency, apart from the tiny thermal hysteresis around $T_{N}$. The downward trend of both FC $M(T)$ curves below $75 \mathrm{~K}$ possibly arises from the negative internal field on the $\mathrm{Yb}^{3+}$ moments (opposite to the $H$ ), exerted by the weak ferromagnetic (WFM) component of canted $\mathrm{Cr}^{3+}$ moments in the AFM spin structure, as discussed in details in our
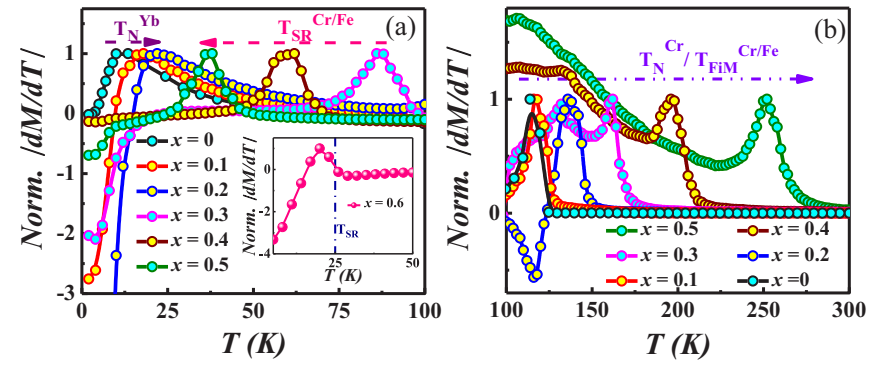

FIG. 3. Derivative of $M$ with respect to $T$ for the determination of $T_{N}{ }^{Y b}$ and $T_{S R}{ }^{C r / F e}$, and $T_{N}{ }^{C r} / T_{F i M}{ }^{C r / F e}$ for $x=0-0.5$ samples in the temperature range (a) 2-100 K, and (b) 100-300 K, respectively. Inset of (a) shows the same spectra for $x=0.6$ in the temperature range $5-50 \mathrm{~K}$.

previous report [27]. In the ZFC protocol, the easy axis of the $\mathrm{Yb}^{3+}$ moments start to align along the field direction when $H$ is applied at the lowest $T$, resulting the net positive $M$. On heating above $T_{N}{ }^{Y b}$, the random orientation of $\mathrm{Yb}^{3+}$ moments in the paramagnetic state leads to a decrease in the net $M$ with increasing temperature. The net $M$ eventually attains zero value at about $24 \mathrm{~K}$ when the $\mathrm{Yb}^{3+}$ sublattice magnetization value becomes equal to that of the $\mathrm{Cr}^{3+}$ sublattice (antiparallel orientation). Above $24 \mathrm{~K}$, a broad minimum centered around $75 \mathrm{~K}$ is found, and beyond that $M$ starts to increase as $\mathrm{Cr}^{3+}$ moments tend to align along the direction of $H$. Finally, the $M$ becomes positive near $T_{N}$. In addition, the ZFC and FC $M(T)$ data measured at $H=1000$ Oe for $x=0$ are also presented in Fig. S3 in the SM [52]. It is observed that ZFC magnetization becomes positive throughout the temperature range under 1000 Oe magnetic field, whereas FC magnetization having $T_{\text {comp }}=10 \mathrm{~K}$ still shows a negative value. As the magnitude of the internal field on $\mathrm{Yb}^{3+}$ moments (acting in the opposite direction of $H$ ) decreases with the increase of applied magnetic field [27], $T_{\text {comp }}$ is slowly shifted to lower value, and finally, the magnetization reversal phenomenon disappears at higher fields.

Figures 2(b) and 2(c) show the $M-T$ data for $x=0.1$ and 0.2 , respectively. It is quite obvious that magnetic interactions are getting modified with $\mathrm{Fe}$ doping, and as a result, we observe the shifting of magnetic phase transition temperatures towards higher temperatures for $x=0.1$ and 0.2 [Figs. 3(a) and 3(b)]. Here, we have designated the high-temperature (high- $T$ ) magnetic phase transition temperature as $T_{F i M}$ for $x \geqslant 0.1$, the origin of which will be discussed later. Providing the identical value of superexchange coupling parameter $(J)$, the higher value of $T_{F i M}$ for the doped samples (compared to $T_{N}$ of $x=0$ ) can be well understood on the basis of the formula $k_{\mathrm{B}} T_{\mathrm{N}}=4 S(S+1) J$ as the total spin $S$ of $\mathrm{Fe}^{3+}$ ions $\left(t_{2 g}^{3} e_{g}^{2}, S=5 / 2\right)$ is higher than $\mathrm{Cr}^{3+}$ ions $\left(t_{2 g}^{3} e_{g}^{0}, S=3 / 2\right)$. Both the ZFC data display a valleylike feature below $T_{F i M}$, although no MR phenomenon is observed in these samples. This valley-like feature of ZFC $M(T)$ can be interpreted by the similar mechanism as described for $x=0$, provided that the value of $\mathrm{Yb}^{3+}$ sublattice magnetization won't be equal to $\mathrm{Cr}^{3+} / \mathrm{Fe}^{3+}$ sublattice magnetization at any temperature. A similar valleylike feature in ZFC $M(T)$ was recently reported for ferrimagnetic (FiM) double perovskite compound 
$\mathrm{Er}_{2} \mathrm{CoMnO}_{6}$, and the observed feature is explained on the basis of the three-sublattice model [53]. Interestingly, FC $M(T)$ data for both these samples show double compensation points (intersecting the $M=0$ line twice) and the observed $M$ is negative between 2 and $26 \mathrm{~K}$, and 8 and $30 \mathrm{~K}$ for $x=0.1$ and 0.2 , respectively. It is to be noted that the $T_{N}{ }^{Y b}$ is also influenced by the presence of $\mathrm{Fe}^{3+}$ ions and it moderately increases for $x=0.1$ and 0.2 [Fig. 3(a)]. Except the sharp increase in magnetization below the lowest value of $M$, the basic physical insight of such variation in FC $M(T)$ data for $x=0.1$ and 0.2 remains almost the same as that of $x=0$. Finally, $M$ turns positive after crossing the $M=0$ line at $T_{\text {comp2 }}$, which is not seen for $x=0$. The sharp increase in FC $M(T)$ at low- $T$ possibly arises from the modified $\mathrm{Yb}^{3+}-\mathrm{Cr}^{3+} / \mathrm{Fe}^{3+}$ interactions in the doped samples, which play a crucial role in determining the direction and magnitude of total $M$ below the rare-earth ordering temperature [Fig. 3(a)].

On the other hand, an extra magnetic phase transition (very sharp in nature) in both ZFC and FC $M(T)$ data is observed for $x=0.3-0.6$ apart from the high- $T$ FiM transition, which introduces significant thermal hysteresis between the FCC and FCH data over a wide range of temperature [Figs. 2(d) $-2(\mathrm{~g})]$. The thermal hysteresis indicates the firstorder nature of this magnetic transition. However, in the earlier investigations on perovskite rare-earth orthochromites and orthoferrites, this kind of sharp magnetic phase transition has been attributed to the spin reorientation (SR) of transition metal (TM) ions [18,19,38,54-56]. Accordingly, we have also defined this sharp magnetic transition as the SR of $\mathrm{Cr}^{3+} / \mathrm{Fe}^{3+}$ ions and the corresponding temperature as $T_{S R}$. It is evident that the direction of the magnetic easy axis (MEA) of TM ions suddenly changes around this particular temperature. The magnitude of $M$ again starts to increase below $T_{S R}$ due to the ordering of $\mathrm{Yb}^{3+}$ moment along with the $\mathrm{Yb}^{3+}-\mathrm{Cr}^{3+} / \mathrm{Fe}^{3+}$ interaction. The $T_{S R}$ continuously decreases with the increases in the Fe-doping level at the $\mathrm{Cr}$-site of pristine $\mathrm{YbCrO}_{3}$, which is in contrast to the increasing nature of $T_{F i M}$ with doping [Figs. 3(a) and 3(b)]. Nevertheless, no MR phenomenon is noticed in any measurement protocol for these samples. It is to be mentioned here that $T_{F i M}$ lies above room temperature for $x=0.6\left(T_{F i M}>320 \mathrm{~K}\right)$. In addition, we also assume that the sharp peak below $T_{F i M}$ in FCC $M(T)$ for $x=0.2$ might be an indication of SR of TM ions, where $T_{S R}$ is higher than that of $x=0.3$ [Fig. 2(c)]. To check the feasibility of SR phenomenon and thermal hysteresis at high magnetic field, we have measured ZFC and FC $M(T)$ at different fields (100 Oe, $500 \mathrm{Oe}, 1 \mathrm{kOe}$, and $10 \mathrm{kOe}$ ) for $x=0.5$ (Figs. S4(a)-S4(d) in the SM [52]). When the strength of the $H$ is gradually increased, the thermal hysteresis between FCC and FCH $M(T)$ curves decreases in accordance with that, and eventually, all three $M(T)$ curves lie on the same line for $H \geqslant 10$ kOe. Although the SR phenomenon remains unscathed for $H \leqslant$ $10 \mathrm{kOe}$, the change in the magnetization $(\Delta M)$ just below $T_{S R}$ significantly reduces with the increase of $H$. This signifies that the high- $H$ prevents the TM ions from reorienting their easy axis of magnetization.

To understand the nature of magnetic phases around the high- $T$ onset of magnetic orderings, we interpret the temperature dependent inverse susceptibility $\chi^{-1}(T)$ just above these ordering temperatures for $x=0,0.1,0.3$, and 0.4 samples,
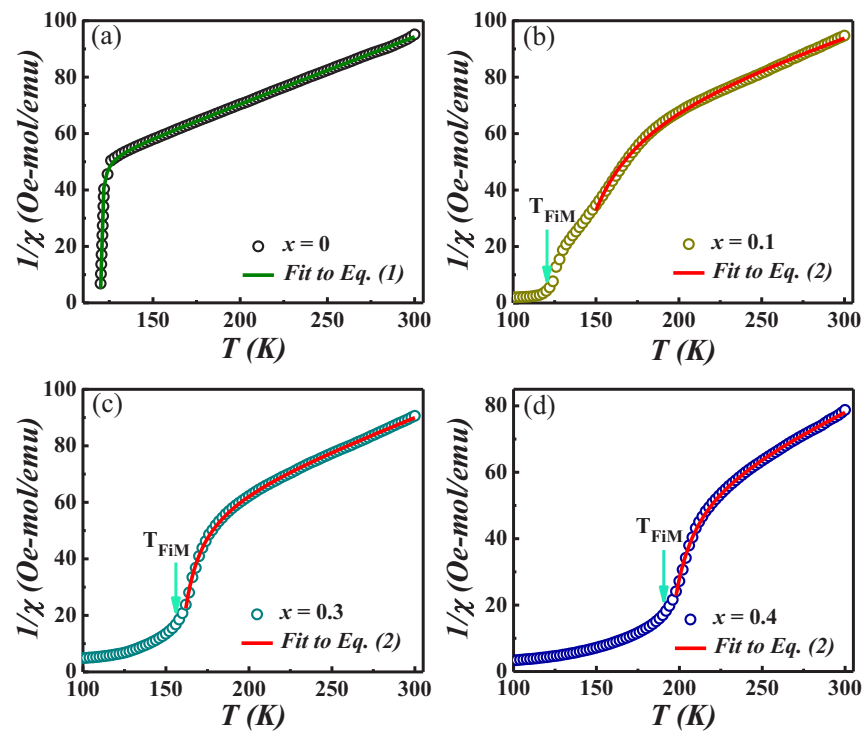

FIG. 4. Inverse magnetic susceptibility $(1 / \chi)$, calculated using $100 \mathrm{Oe} \mathrm{FCH} \mathrm{data,} \mathrm{as} \mathrm{a} \mathrm{function} \mathrm{of} \mathrm{temperature} \mathrm{for} \mathrm{(a)} x=0$, (b) $x=0.1$, (c) $x=0.3$, and (d) $x=0.4$. Solid lines in (a) and (b)-(d) are fit to the Eqs. (1) and (2), respectively.

as displayed in Figs. 4(a)-4(d), respectively. The sharp fall in $\chi^{-1}(T)$ near $T_{N}$ for $x=0$ sample possibly originates from the antisymmetric exchange interaction between $\mathrm{Cr}^{3+}$ spins of canted AFM spin structure, and the $\chi^{-1}(T)$ above $T_{N}$ has been fitted with the modified Curie-Weiss equation, originally proposed by DM $[57,58]$

$$
\chi=\frac{C}{T-\Theta} \frac{T-T_{0}}{T-T_{N}},
$$

where $C, \Theta, T_{0}$, and $T_{N}$ is the Curie constant, the Weiss temperature, a fitted parameter, and the Néel temperature of Cr ordering, respectively [Fig. 4(a)]. The term $\frac{T-T_{0}}{T-T_{N}}$ in Eq. (1) determines the sharp fall in $\chi^{-1}(T)$ near $T_{N}$, provided that the value of $T_{0}$ is close to $T_{N}$. Thus, the well-fitted $\chi^{-1}(T)$ above $T_{N}$, and almost similar value of $T_{0}(=119.4 \mathrm{~K})$ and $T_{N}(=119.9 \mathrm{~K})$ confirm the antisymmetric DM interaction between canted $\mathrm{Cr}^{3+}$ spins, which, in particular, brings the weak ferromagnetism in the $x=0$ sample. In spite of the sharp fall near the high- $T$ onset of magnetic orderings, a different behavior of the $\chi^{-1}(T)$ is identified for Fe-doped samples. For instance, the $\chi^{-1}(T)$ for $x=0.1$ sample reveals hyperbolic kind of feature well above the high- $T$ magnetic ordering, which tends to be more profound in the case of $x=0.3$ and 0.4 samples, as displayed in Figs. 4(b)-4(d). Even though $\chi^{-1}(T)$ above the transition temperature for $x=0.1,0.3$, and 0.4 samples can be fitted using Eq. (1), a significant deviation from the expected $T_{N} \approx T_{0}$ inhibits us to interpret the data employing DM interaction model. However, according to the mean-field theory, the hyperbolic behavior of $\chi^{-1}(T)$ above the transition temperature is a characteristic of FiM systems and better described by the Néel's expression [59]

$$
\chi^{-1}(T)=\frac{T-\Theta}{C}-\frac{\xi}{T-\Theta^{\prime}},
$$


(a)
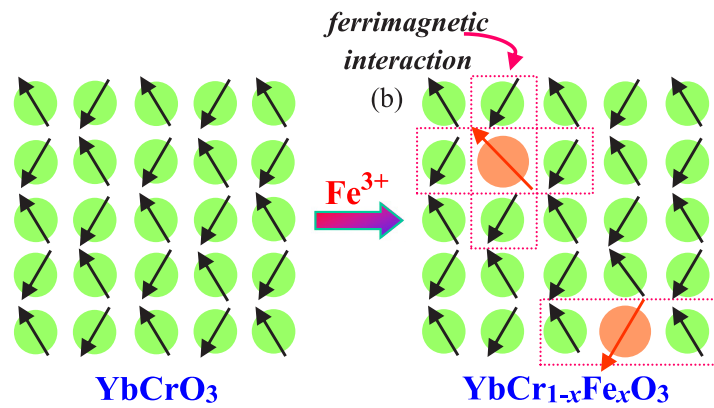

- Cr ions;

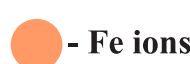

; $\uparrow-\mathrm{Cr}^{3+}$ spins; $\uparrow-\mathrm{Fe}^{3+}$ spins

FIG. 5. Macroscopic model for the realization of FiM ground state in the doped samples. (a) Canted spin-arrangement in the AFM structure of $\mathrm{YbCrO}_{3}$, producing WFM component. (b) Displays the emergence of short-range ferrimagnetic interaction between $\mathrm{Cr}^{3+}$ and $\mathrm{Fe}^{3+}$ ions in the canted AFM structure of the doped samples. The magnitude of the total spin of $\mathrm{Fe}^{3+}$ ion is represented by the larger arrow.

where the first term represents the high- $T$ asymptotic behavior that has a simple Curie-Weiss form, and the latter term represents the low- $T$ hyperbolic behavior near the FiM transition [60-62]. Here, $\Theta^{\prime}$ and $\xi$ are fitting parameters and find their origins in the two-sublattice model of FiM [59]. The red line in Figs. 4(b)-4(d) depicts the fitted results of Eq. (2) to $\chi^{-1}(T)$ above transition temperature. An excellent fit thus confirms the FiM nature of magnetic phases for $x=0.1$, 0.3 , and 0.4 samples. Hence, the magnetic ground state has transformed from canted AFM to FiM with Fe doping at $\mathrm{Cr}$ site of $\mathrm{YbCrO}_{3}$. A feasible mechanism for the appearance of FiM ground state is schematically shown in Fig. 5. The canted AFM spin arrangement of $\mathrm{Cr}^{3+}$ ions in $\mathrm{YbCrO}_{3}$ is depicted in Fig. 5(a). However, the random substitution of $\mathrm{Cr}$ by Fe leads to some short-range FiM interaction between the $\mathrm{Cr}^{3+}$ and $\mathrm{Fe}^{3+}$ spins, as indicated by the dotted box in Fig. 5(b), for lower Fe-doped samples. The strength of the FiM interaction becomes even stronger for higher Fe-doped samples due to the presence of moderate $\mathrm{Fe}^{3+}$ ions in the vicinity of $\mathrm{Cr}^{3+}$ ions, and as a result, we observe a distinct hyperbolic-kind of feature above the high- $T$ magnetization anomalies.

\section{Magnetic structure}

In order to have deeper understanding of the SR phenomenon and the magnetic structures in the different magnetically ordered regions, we have performed temperature dependent NPD measurements for $x=0$ and 0.4 samples. NPD patterns were recorded in the temperature range 3$300 \mathrm{~K}$ for both samples. A typical Rietveld refinement of the observed NPD data at $3 \mathrm{~K}$ is shown in Fig. 6 for $x=0$ and 0.4 samples. As $x=0.4$ sample is expected to exhibit SR around $70 \mathrm{~K}$, we also examine the NPD pattern at $140 \mathrm{~K}$ to look for the magnetic structure before the SR transition. The magnetic structure for $x=0$ was modeled as per Ref. [63] and an excellent compliance with the starting model has been observed. In the case of $x=0.4$, the spin-structure above the SR transition temperature can be represented by Fig. 7(a) (at $140 \mathrm{~K}$ ) and described using $G_{x}$ and $G_{y}$ notations for $\mathrm{Yb}$ and

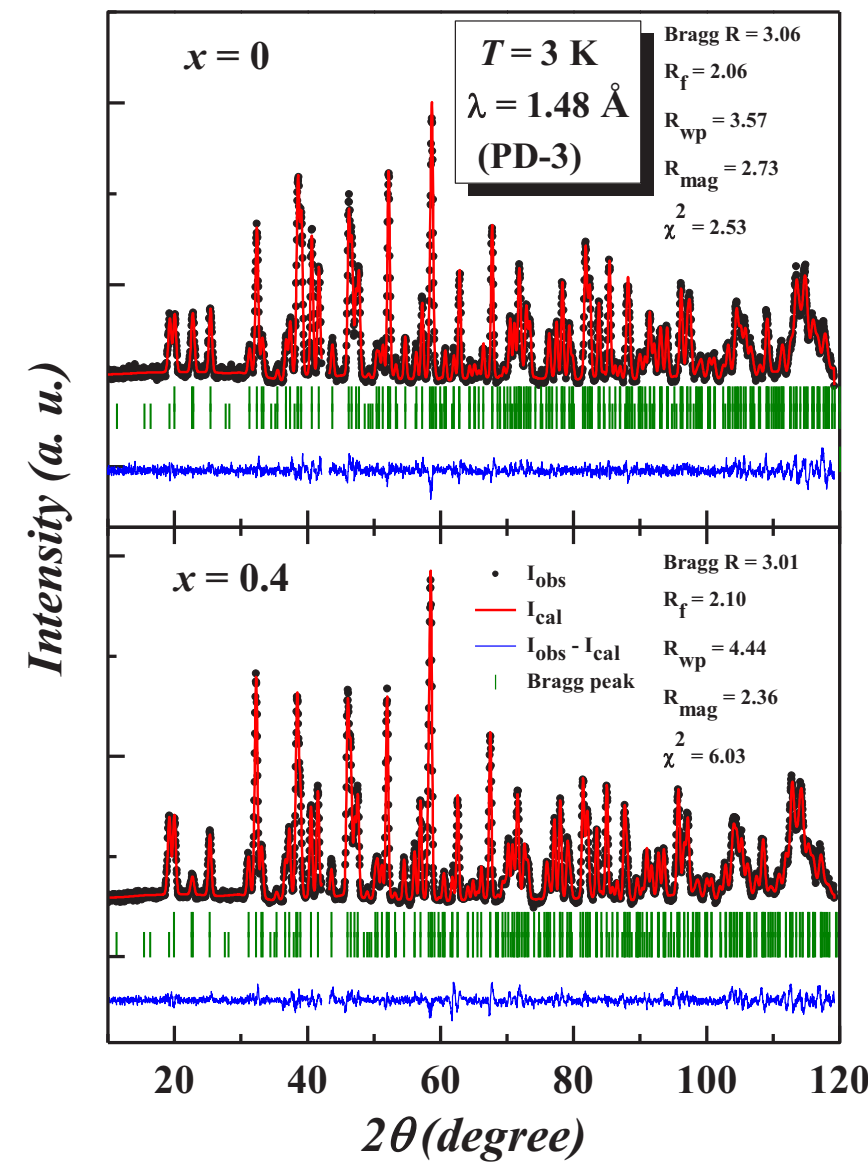

FIG. 6. Observed (solid black circles) and calculated (solid red line) NPD patterns at $T=3 \mathrm{~K}$ for $x=0$ (upper panel) and $x=0.4$ (lower panel) samples. The blue solid line represents the difference between the observed and calculated patterns. The vertical ticks in each panel indicate the position of allowed nuclear (upper row) and magnetic (bottom row) Bragg peaks.

$\mathrm{Cr} / \mathrm{Fe}$ moments, respectively. However, the magnetic structure for both these compounds at $3 \mathrm{~K}$ can be represented by Fig. 7(b), where $\mathrm{Yb}$ moments are aligned ferromagnetically along the $x$ direction and can be described using the notation $F_{x}$ as given in Ref. [63]. Similarly, $\mathrm{Cr}$ moments can be expressed by the notation $G_{\mathrm{z}}$. The main point to be highlighted here is that, as seen in Figs. 7(a) and 7(b), the direction of $\mathrm{Cr}$ moments has flipped by $90^{\circ}$ between 3 and 140 K. In Fig. 7(a), both $\mathrm{Yb}$ and $\mathrm{Cr}$ moments are in the $a b$ plane at $140 \mathrm{~K}$ for $x=$ 0.4; whereas, for both $x=0$ and 0.4 [see Fig. 7(b)], the $\mathrm{Cr}$ moments at $3 \mathrm{~K}$ (below SR transition) are aligned along the $c$ axis (or $z$ direction) and $\mathrm{Yb}$ moments are in the $a b$ plane. The data measured at $3 \mathrm{~K}$ alone are shown in Fig. 6; however, NPD measurements for $x=0$ and 0.4 were carried out at various temperatures as seen by the unit-cell parameters plotted in Figs. S5 in the SM [52], showing almost conventional thermal expansion above $T_{N}{ }^{Y b}$ and $T_{S R}$ for $x=0$ and 0.4 , respectively. However, a small negative thermal expansion (NTE) can be seen in the in the lattice constants $a$ and $b$ of $x=0.4$ when the sample is cooled below $T_{S R}$ (Fig. S5(d) in the SM [52]). Thus, the smaller value of NTE $(\sim 0.006 \%)$ below $T_{S R}$ unveils the small spontaneous magnetostriction in the $x=0.4$ 

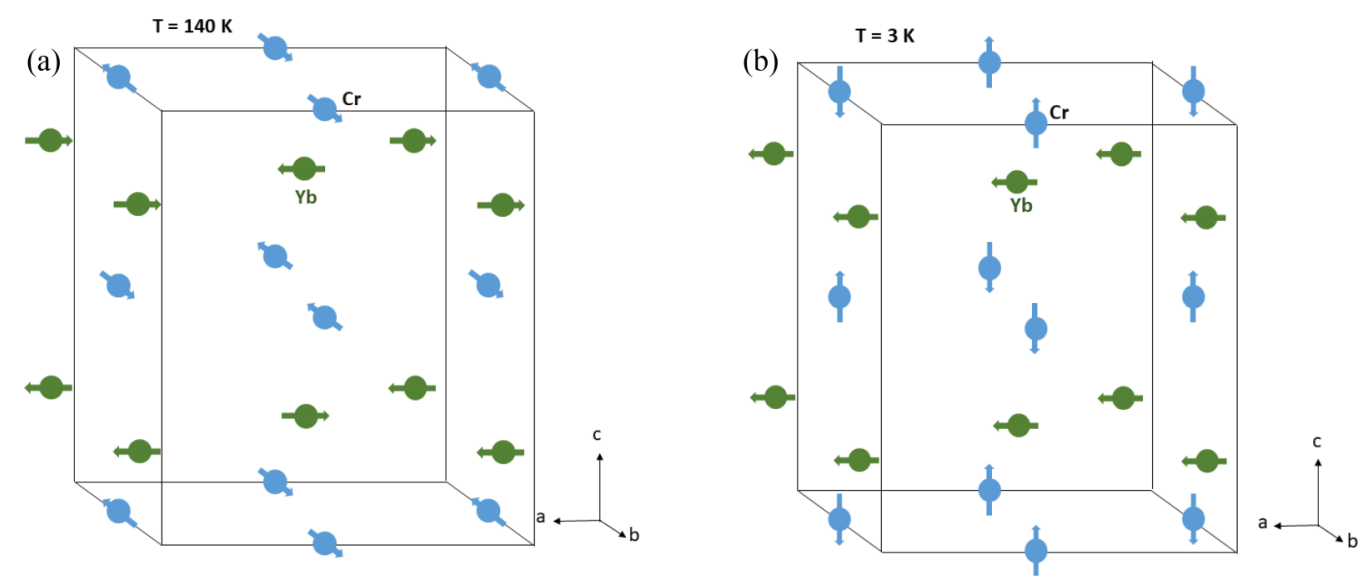

FIG. 7. Magnetic structures of $x=0.4$ at (a) $T=140 \mathrm{~K}$, and (b) $T=3 \mathrm{~K}$, where $\mathrm{Yb}^{3+}$ and $\mathrm{Cr}^{3+} / \mathrm{Fe}^{3+}$ ions/moments are shown as olive and cyan spheres/arrows, respectively. $\mathrm{O}^{2-}$ anions are not shown here for clarity. The magnetic structure at $T=3 \mathrm{~K}$ for $x=0$ is also similar to $(b)$.

sample, which is one order of magnitude less than the expected value of NTE for the samples having magnetoelastic coupling $[38,43,64]$. Though, we have found a small NTE in our sample, the origin of such NTE still could be ascribed to the magnetoelastic effect generated by the repulsion between the neighboring TM magnetic moments [38].

The temperature dependence of the refined $\mathrm{Yb}$ and $\mathrm{Cr} / \mathrm{Fe}$ moments for $x=0$ and 0.4 are displayed in Figs. 8(a) and 8 (b), respectively. It is observed that the magnetic moments of $\mathrm{Yb}$ and $\mathrm{Cr}$ have disappeared above $120 \mathrm{~K}$, which is close to the observed $T_{N}$ for $x=0$. Interestingly, both $\mathrm{Yb}$ and $\mathrm{Cr}$ magnetic moments show anomaly at about $T_{N}{ }^{Y b}$, below which the value of the $\mathrm{Yb}$ moment suddenly increases due to the ordering of rare-earth $\mathrm{Yb}^{3+}$ ion, whereas the value of $\mathrm{Cr}$ moment decreases. On the other hand, the magnetic moments of $\mathrm{Yb}$ and $\mathrm{Cr} / \mathrm{Fe}$ for $x=0.4$ reduce to nearly zero value at about $T_{F i M}$, and exhibit certain anomalies near $T_{S R}(\sim 70 \mathrm{~K})$. Nonetheless, this anomaly in both $\mathrm{Yb}$ and $\mathrm{Cr} / \mathrm{Fe}$ moments around $T_{S R}$ appears due to the spin reorientation, which is possibly driven by $f-d$ exchange interaction [65]. On cooling below $T_{S R}$, the value of $\mathrm{Yb}$ moment decreases, while the $\mathrm{Cr} / \mathrm{Fe}$ moment increases.

\section{Magnetocaloric effect}

In general, any kind of first-order magnetic phase transition is associated with the corresponding isothermal magnetic entropy change $\left(\Delta S_{M}\right)$, which gives rise to the significant
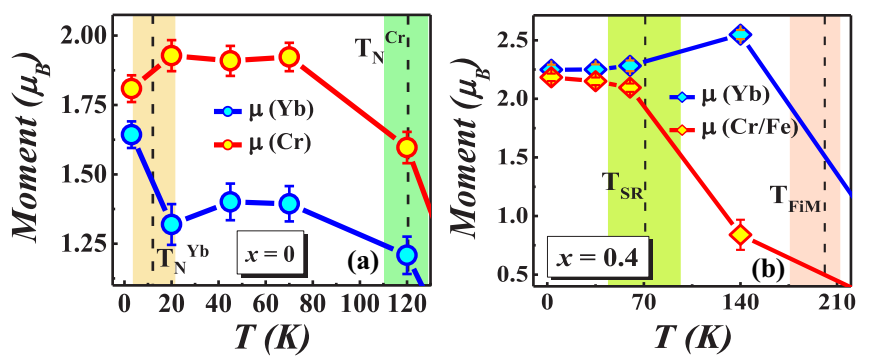

FIG. 8. Temperature variations of the ordered magnetic moment of the $\mathrm{Yb}^{3+}$ and $\mathrm{Cr}^{3+} / \mathrm{Fe}^{3+}$ ion in (a) $x=0$, and (b) $x=0.4$ samples. magnetocaloric effect (MCE) in the material [66-69]. Thus, the first-order SR phenomenon in $x=0.3-0.6$ samples encouraged us to study the MCE through isothermal magnetization measurements. We choose the $x=0.4$ sample for a case study here to explore the value of $\Delta S_{M}$ around this $T_{S R}(=70 \mathrm{~K})$. The value of $\Delta S_{M}$ can be calculated from the isothermal magnetization measurements (indirectly) using the Maxwell's thermodynamic relation:

$$
\Delta S_{M}(T, \Delta H)=\int_{H_{i}}^{H_{f}}\left(\frac{\partial M(T, H)}{\partial T}\right)_{H} d H,
$$

where $H_{i}$ and $H_{f}$ represent the initial and final applied magnetic fields, respectively. The temperature dependent $-\Delta S_{M}$ for the $x=0.4$ sample in the temperature range $10-145 \mathrm{~K}$ at different magnetic field changes is shown in Fig. 9. The enlarged view of $-\Delta S_{M}$ within $50-90 \mathrm{~K}$ for the highest magnetic field changes is shown in the inset of Fig. 9. It is seen that the $x=0.4$ sample reveals a weak anomaly in

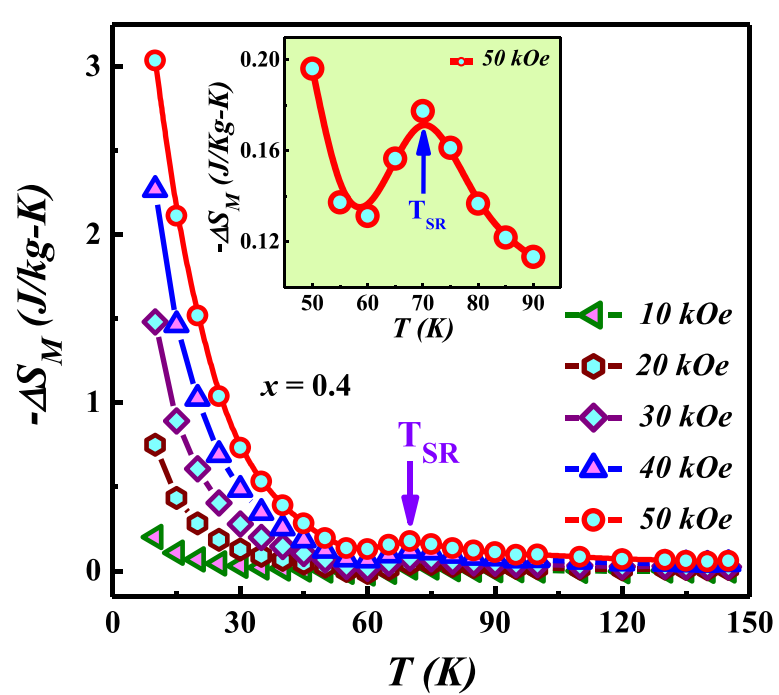

FIG. 9. Temperature dependence of magnetic entropy change $-\Delta S_{M}$ under the applied magnetic field changing from 10 to $50 \mathrm{kOe}$ for $x=0.4$. 

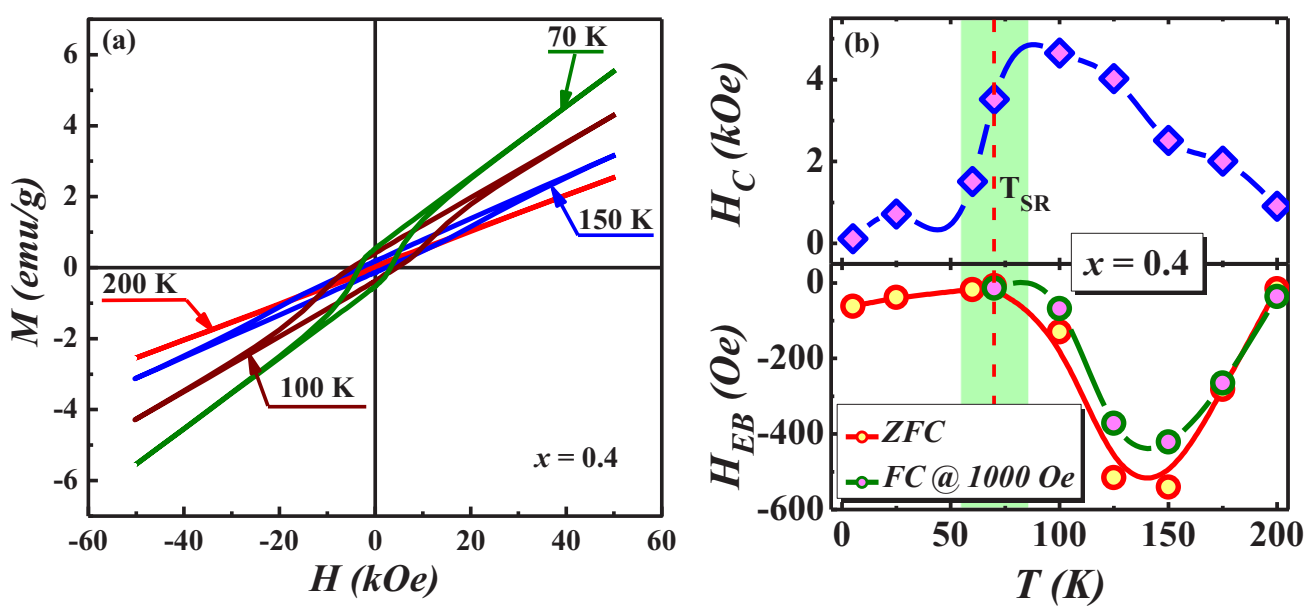

FIG. 10. (a) Isothermal magnetization $(M)$ as a function of magnetic field $(H)$ within $\pm 50 \mathrm{kOe}$ under ZFC mode at four different temperatures for $x=0.4$. (b) Shows the thermal profiles of the estimated coercive field $\left(H_{C}\right)$ (upper panel) and exchange bias field $\left(H_{E B}\right)$ (lower panel) for $x=0.4$. The red dashed line is guide to the eye for the temperature corresponding to SR transition of $x=0.4$.

$-\Delta S_{M}$ at $T_{S R}$ and the magnitude of $-\Delta S_{M}$ is very small. The magnitude further increases with decreasing temperature and increasing the strength of the magnetic field. In contrast, no such anomalous behavior of $-\Delta S_{M}$ is observed in $x=0$ sample around $70 \mathrm{~K}$ [27]. Although, a significant change in $-\Delta S_{M}$ value near the $T_{S R}$ has been predicted theoretically for single-crystalline samples [68], we do not observe such large changes in $-\Delta S_{M}$ for polycrystalline $x=0.4$ sample. As single-crystalline materials provide highly preferred crystallographic orientation, they also exhibit anisotropic behavior in the magnetocaloric effect. Thus, a significant contribution from magnetic anisotropy towards the changes in $-\Delta S_{M}$ along with the possible changes of exchange energy (due to magnetic ordering) can be theoretically expected for the single crystals, having preferred crystallographic orientation. On the other hand, polycrystalline materials consist of several grains with different crystallographic orientations, and it is obvious that the contribution from magnetic anisotropy towards the changes in $-\Delta S_{M}$ will be an average value over all the grains, which is indeed lower than the single-crystalline samples. As a result, we observe the small changes in $-\Delta S_{M}$ for the polycrystalline $x=0.4$ sample near the $T_{S R}$, which is in contrast to the theoretical prediction on single crystals.

\section{E. Magnetic field dependence of magnetization}

In order to view further consequences of this SR phenomenon, we have carried out the isothermal magnetic field dependent magnetization $M(H)$ measurements for the $x=0.4$ sample. The isothermal ZFC $M-H$ curves for $x=0.4$ at four different temperatures $(T=70,100,150$, and $200 \mathrm{~K})$ are shown in Fig. 10(a). All the $M-H$ curves show appreciable hysteretic behavior in the low- $H$ region, whereas almost linear variation of $M$ with $H$ is observed in the high- $H$ region. This indicates the presence of both FM and AFM phases in the sample below $T_{F i M}$. We deduce the value of the coercive field $\left(H_{C}\right)$ from the $M-H$ curve at different fixed temperatures and the variation of $H_{C}$ with $T$ is displayed in the upper panel of Fig. 10(b). The value of $H_{C}$ starts to increase with decreases in temperature below $T_{F i M}$ and exhibits a certain downfall around $T_{S R}$, which implies that the changes in the direction of MEA (due to the SR transition) significantly reduces the strength of the required $H$ to demagnetize the sample. Strikingly, an asymmetry and shifting along the negative $H$ axis is found for all the ZFC $M-H$ below $180 \mathrm{~K}$, which dictates the exchange bias (EB) effect in the system. The asymmetric nature of ZFC $M-H$ loop at a selected temperature $(\sim 175 \mathrm{~K})$ in enlarged scale is shown in Fig. S6 [52]. We have precisely evaluated the value of EB field $\left(H_{E B}\right)$ in ZFC mode at different temperatures in order to investigate the $T$ variation of $H_{E B}$ as depicted in the lower panel of Fig. 10(b). The FC $M$ - $H$ curve at the same fixed temperatures were also measured after cooling the sample in the presence of $H=1000$ Oe from $300 \mathrm{~K}$ to check the conventional EB behavior of $x=0.4$, and the variation of $H_{E B}$ as function of $T$ is shown in the lower panel of Fig. $10(\mathrm{~b})$. In both the cases, $H_{E B}$ reaches the maximum value around $150 \mathrm{~K}$, which is well below the $T_{F i M}$, and decreases to almost zero value near the $T_{S R}(=70 \mathrm{~K})$. The magnitude of $H_{E B}$ again slightly increases below $T_{S R}$. As the direction of MEA of TM ions changes, the total FM component is inherently affected, and as a result, $H_{E B}$ shows an anomalous behavior around the $T_{S R}$. It should be noted that the value of $H_{E B}$ is less in the case of the FC mode than the ZFC mode, which presumably arises due to the depletion of FM component in the presence of moderate $H$ during the FC process.

To examine the rationality of this anomalous behavior of $H_{E B}$ around $T_{S R}$, we have additionally performed isothermal $M(H)$ measurements for $x=0.5$ at different temperatures below the $T_{F i M}$. Figure 11(a) depicts the isothermal ZFC $M(H)$ curves at $T=40,46,50$, and $70 \mathrm{~K}$. It is clear that hysteretic loop opening notably reduces below $50 \mathrm{~K}$. Nevertheless, to show the thermal variation of $H_{C}$ and ZFC exchange bias field $\left(H_{\mathrm{ZEB}}\right)$, we plot the value of $H_{C}$ and $H_{\mathrm{ZEB}}$ as a function of temperature $(T)$ in Figs. 11(b) and 11(c), respectively. A clear change in the slope of $H_{C}$ vs $T$ curve is found at around $T_{S R}$, while the $H_{\mathrm{ZEB}}$ vs $T$ curve reveals a perceptible dip at $T_{S R}$ $(=48 \mathrm{~K}$ for $x=0.5)$. These facts further elucidate the crucial role played by the SR of TM ions to explain the observed temperature $(T)$ dependent magnetization $(M)$ behavior of $x=0.3-0.6$ samples. 

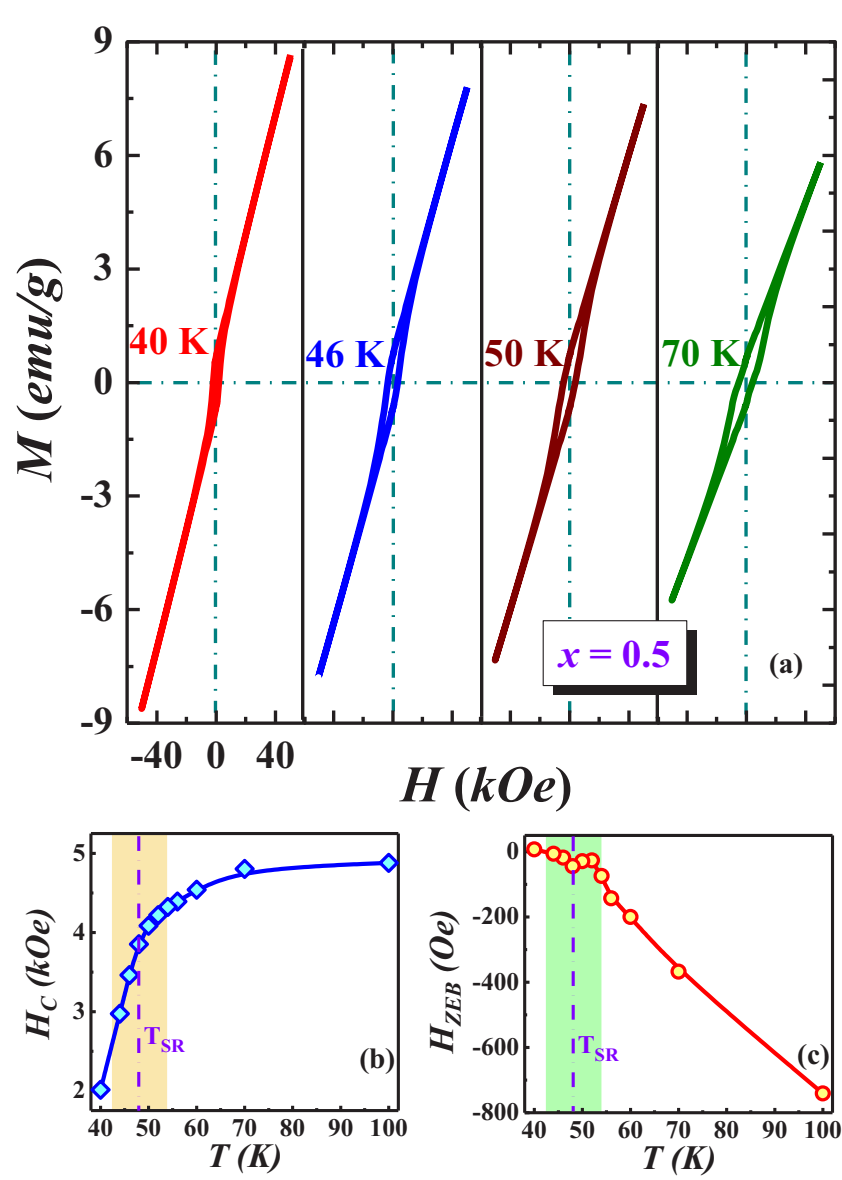

FIG. 11. (a) ZFC $M-H$ loops measured at four different temperatures within $\pm 50 \mathrm{kOe}$ around the SR transition temperature $\left(T_{S R}\right)$ for $x=0.5$. (b) and (c) show the temperature variations of $H_{C}$ and ZFC exchange bias field $\left(H_{\mathrm{ZEB}}\right)$ for $x=0.5$, respectively. The violet dash-dotted line in both (b) and (c) indicates the $T_{S R}$ of $x=0.5$.

\section{F. Dielectric properties and magnetostriction}

In order to probe whether there is any magnetoelectric coupling around both the $T_{S R}$ and $T_{F i M}$, we have performed the temperature-dependent dielectric measurements for $x=$ 0.3 and 0.4 samples at different frequencies $(f=157 \mathrm{~Hz}, 1$ $\mathrm{kHz}$, and $10 \mathrm{kHz})$. The dielectric constant $\left(\varepsilon_{r}\right)$ monotonously increases with increase of temperature, and no maximum is observed in dielectric spectra within the investigated temperature range 5-290 K for $x=0.3$ [Fig. 12(a)] and 140-300 K for $x=0.4$ [Fig. 12(c)]. The value of $\varepsilon_{r}$ at a particular temperature decreases with the increase of frequency. However, a distinct anomaly around $T_{F i M}$ is noted in the derivative of dielectric constant with respect to temperature [see the insets in Figs. 12(a) and 12(c)]. This finding thus establishes the presence of magnetoelectric coupling phenomenon in both $x=0.3$ and 0.4 samples. In addition, the dielectric loss $(\tan \delta)$ spectra for $x=0.3$ and 0.4 are shown in Figs. 12(b) and 12(d), respectively. The same spectra for $x=0.3$ are also displayed in the expanded scale (100-225 K) in the inset of Fig. 12(b). A shoulderlike feature can be seen in $157 \mathrm{~Hz}$ loss $(\tan \delta$ ) spectra of $x=0.3$ at about $T_{F i M}$, which exhibits significant frequency dispersion, and the shoulder in the loss spectra is shifted toward the higher temperature with the increase of frequency. This kind of frequency dispersion feature of the loss spectra is a characteristic of the ferroelectric relaxor material [70], and relaxor behavior might have originated from the magnetic frustration related to the competing AFM and FiM interactions, accompanied by spin-lattice coupling. Note that the $\varepsilon_{r}$ spectra of both $x=0.3$ and 0.4 , and the loss spectra of $x=0.4$ also exhibit similar frequency dispersion. On the other hand, the derivative of loss spectra in the inset of Fig. 12(d) reveals a pronounced anomaly around the ferrimagnetic ordering for $x=0.4$. Though we observe the ferroelectric relaxor behavior and magnetoelectric coupling around the ferrimagnetic ordering temperature in the perovskite phases of $\mathrm{YbCr}_{1-x} \mathrm{Fe}_{x} \mathrm{O}_{3}$, similar results have been noticed in earlier studies for ferromagnetic double perovskites $\mathrm{La}_{2} \mathrm{NiMnO}_{6}$ [71] and $\mathrm{La}_{2} \mathrm{CoMnO}_{6}$ [72].

To find out the origin of magnetoelectric coupling in these samples, the temperature dependent thermal expansion $(\Delta L / L)$ measurements in zero-field and with applied field are performed for $x=0.3$, as shown in Fig. 12(e). Two dips (weak in nature) are found in the derivative spectra of $(\Delta L / L)$ at $H=0 \mathrm{kOe}$ around the transition temperatures $T_{F i M}$ and $T_{S R}$, respectively [as indicated by arrows, see the inset in Fig. 12(e)]. The temperature dependence of magnetostriction, which is in general defined as $((\Delta L(0)-\Delta L(H)) / L)$, for the $x=0.3$ sample is also shown in Fig. 12(f), revealing an anomalous behavior around $T_{F i M}$. Further, the magnetostriction shows a negative to positive crossover with decreasing temperature, and consequently, the zero value around $T_{S R}$. This observation suggests that magnetostriction seems to play a pivotal role in the slight changes of dielectric constant around the FiM ordering temperature, i.e., in magnetoelectric coupling phenomenon. The low temperature peak around $50 \mathrm{~K}$ in the magnetostriction data possibly arises due to the $\mathrm{Yb}^{3+}-\mathrm{Cr}^{3+} / \mathrm{Fe}^{3+}$ interaction, as the rare-earth $\mathrm{Yb}^{3+}$ ions also start to engage in magnetic interaction below this temperature.

In contrast, both $\varepsilon_{r}(T)$ and $\tan \delta(T)$ do not exhibit a notable anomaly around $T_{S R}(\sim 90 \mathrm{~K}$ for $x=0.3)$, although a significant magnetoelectric coupling is expected to be seen considering the first-order nature of SR transition. The absence of possible magnetoelectric coupling in both $\varepsilon_{r}$ and tan $\delta$ around $T_{S R}$ is in sharp contrast to that reported for other perovskite orthochromites such as $\mathrm{ErCrO}_{3}$ [19] and $\mathrm{SmCrO}_{3}$ [18], which is likely due to the zero value of magnetostriction around $T_{S R}$ as shown in the temperature dependent magnetostriction data [Fig. 12(f)].

\section{SUMMARY AND CONCLUSION}

To summarize, we have investigated $\mathrm{YbCr}_{1-x} \mathrm{Fe}_{x} \mathrm{O}_{3}(0 \leqslant$ $x \leqslant 0.6$ ) with synchrotron $\mathrm{x}$-ray powder diffraction, neutron powder diffraction, as well as temperature and magnetic field dependent magnetization and dielectric measurements. Our high-resolution synchrotron x-ray diffraction analysis reveals that there exists no miscibility gap over the compositional range, and the oxidation states of $\mathrm{Cr}$ and $\mathrm{Fe}$ remain unaltered. From temperature dependent magnetization study, a monotonous increase of magnetic ordering temperature is found, whereas precise analysis of the inverse susceptibility data unfolds that the magnetic ground state is transformed 

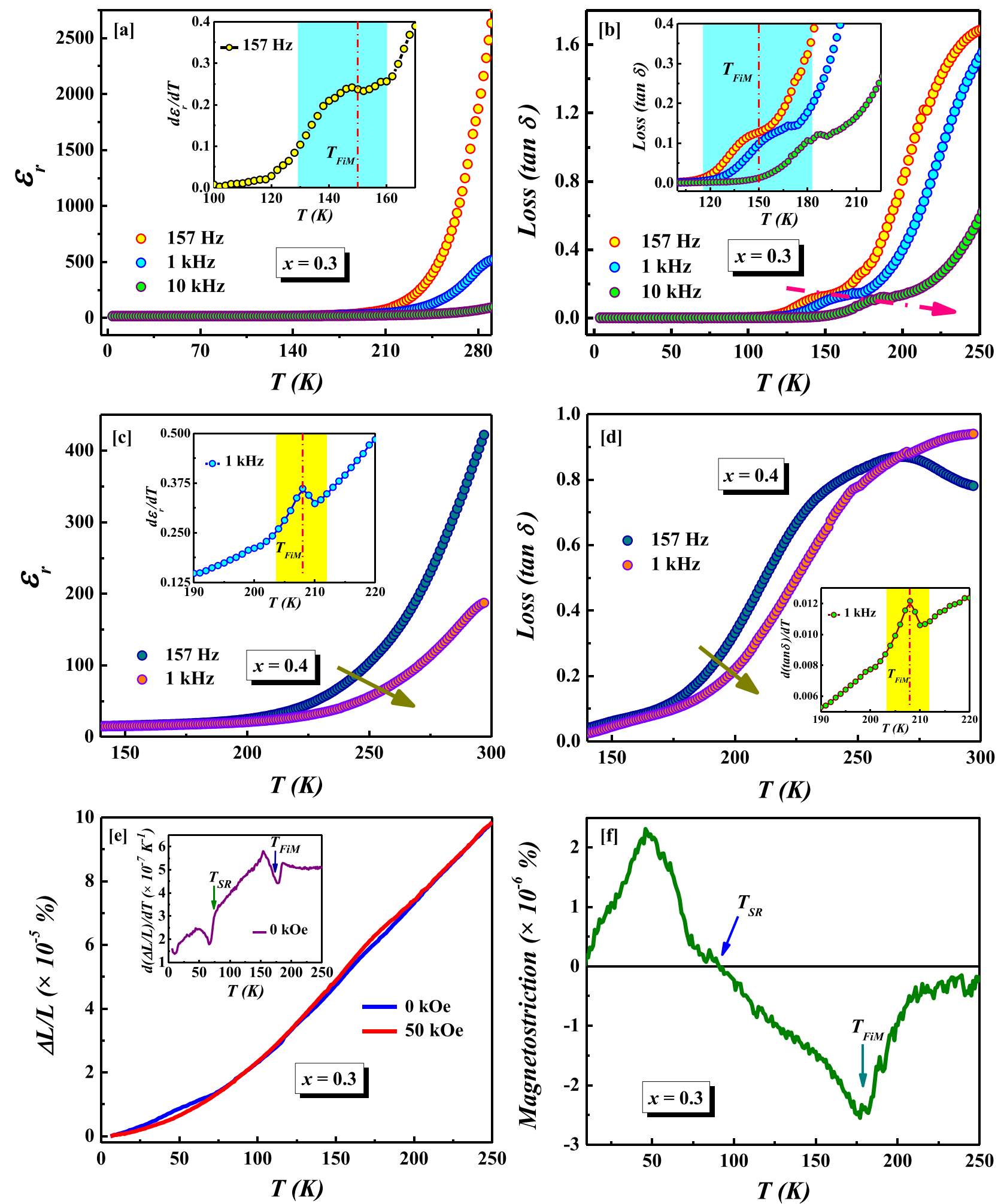

FIG. 12. Temperature $(T)$ dependent dielectric constant $\left(\varepsilon_{r}\right)$ for (a) $x=0.3$, and (c) $x=0.4$ samples recorded at different applied frequencies ( $f=157 \mathrm{~Hz}, 1 \mathrm{kHz}$, and $10 \mathrm{kHz}$ ). Insets show the derivative of the dielectric constant as a function temperature at (a) $f=157 \mathrm{~Hz}$ for $x=0.3$, and (c) $f=1 \mathrm{kHz}$ for $x=0.4$. (b), (d) Temperature dependence of the dielectric loss (tan $\delta$ ) of $x=0.3$, and 0.4 for different frequencies, respectively. Inset in (b) displays the zoomed-in view of the dielectric loss spectra for $x=0.3$ in the temperature region $100-225 \mathrm{~K}$. Inset in (d) shows the derivative of $\tan \delta$ as a function temperature at $f=1 \mathrm{kHz}$ for $x=0.4$. (e) Thermal variations of $\Delta L / L$ in zero-field and an applied field $H=50 \mathrm{kOe}$ for $x=0.3$. Inset shows the derivative spectra $[d(\Delta L / L) / d T]$ of zero-field data. (f) Depicts the temperature dependent magnetostriction $[(\Delta L(0)-\Delta L(H)) / L]$ for $x=0.3$. 
into ferrimagnetic $(x \geqslant 0.1)$ from canted antiferromagnetic $(x=0)$ with Fe doping. The most notable observation is that $x \geqslant 0.3$ samples show a kind of magnetic transition related to the spin reorientation (SR) of $\mathrm{Cr}^{3+} / \mathrm{Fe}^{3+}$ ions, which is further corroborated through the temperature dependent NPD study for $x=0.4$.

Interestingly, the temperature dependence of magnetic entropy change $\left(\Delta S_{M}\right)$, coercivity $\left(H_{C}\right)$, and both ZFC and FC exchange bias field $\left(H_{E B}\right)$ exhibit a significant anomaly at about $T_{S R}$, providing useful insights for the realization of first-order nature of the SR transition and a sudden change in the direction of magnetic easy axis below this transition. In addition, a possible magnetoelectric coupling along with the ferroelectric relaxorlike behavior have been established around the ferrimagnetic ordering temperature for $x=0.3$ and 0.4 samples. Finally, we predict that a similar kind of magne- toelectric coupling and relaxor ferroelectric behavior might be inevitable in the other samples of this series where hightemperature ferrimagnetic phase exists. The observation of magnetoelectric coupling as well as the ferroelectric relaxor behavior around high- $T$ ferrimagnetic ordering and exchange bias effect show these kinds of materials as a promising candidate for technological applications.

\section{ACKNOWLEDGMENTS}

Portions of this research were carried out at the light source PETRAIII of DESY, a member of the Helmholtz Association (HGF). S.K.D. gratefully acknowledges the financial support by the Department of Science \& Technology (Government of India) within the framework of the India@DESY collaboration (Proposal ID: I-20170567).
[1] T. Kimura, T. Goto, H. Shintani, K. Ishiazaka, T. Arima, and Y. Tokura, Nature (London) 426, 55 (2003).

[2] S.-W. Cheong and M. Mostovoy, Nat. Mater. 6, 13 (2007).

[3] D. I. Khomskii, J. Magn. Magn. Mater. 306, 1 (2006).

[4] D. I. Khomskii, Physics 2, 20 (2009).

[5] H. Katsura, N. Nagaosa, and A. V. Balatsky, Phys. Rev. Lett. 95, 057205 (2005).

[6] T. Kimura, Annu. Rev. Mater. Res. 37, 387 (2007).

[7] Y. Tokunaga, S. Iguchi, T. Arima, and Y. Tokura, Phys. Rev. Lett. 101, 097205 (2008).

[8] A. K. Zvezdin and A. A. Mukhin, JETP Lett. 88, 505 (2008).

[9] Y. Tokunaga, N. Furukawa, H. Sakai, Y. Taguchi, T. Arima, and Y. Tokura, Nat. Mater. 8, 558 (2009).

[10] Y. Tokura and S. Seki, Adv. Mater. 22, 1554 (2010).

[11] A. Stroppa, M. Marsman, G. Kresse, and S. Picozzi, New J. Phys. 12, 093026 (2010).

[12] S. Dong, J.-M. Liu, S.-W. Cheong, and Z. Ren, Adv. Phys. 64, 519 (2015).

[13] S. K. Upadhyay, P. L. Paulose, and E. V. Sampathkumaran, Phys. Rev. B 96, 014418 (2017).

[14] S. H. Chun, Y. S. Chai, B.-G. Jeon, H. J. Kim, Y. S. Oh, I. Kim, H. Kim, B. J. Jeon, S. Y. Haam, J.-Y. Park, S. H. Lee, J.-H. Chung, J.-H. Park, and K. H. Kim, Phys. Rev. Lett. 108, 177201 (2012).

[15] T. D. Sparks, M. C. Kemei, P. T. Barton, R. Seshadri, E.-D. Mun, and V. S. Zapf, Phys. Rev. B 89, 024405 (2014).

[16] M. Staruch, G. Lawes, A. Kumarasiri, L. F. Cotica, and M. Jain, Appl. Phys. Lett. 102, 062908 (2013).

[17] S. Mukherjee, A. Dönni, T. Nakajima, S. Mitsuda, M. Tachibana, H. Kitazawa, V. Pomjakushin, L. Keller, C. Niedermayer, A. Scaramucci, and M. Kenzelmann, Phys. Rev. B 95, 104412 (2017).

[18] B. Rajeswaran, D. I. Khomskii, A. K. Zvezdin, C. N. R. Rao, and A. Sundaresan, Phys. Rev. B 86, 214409 (2012).

[19] K. R. S. PreethiMeher, A. Wahl, A. Maignan, C. Martin, and O. I. Lebedev, Phys. Rev. B 89, 144401 (2014).

[20] Y. Su, J. Zhang, Z. Feng, L. Li, B. Li, Y. Zhou, Z. Chen, and S. Cao, J. Appl. Phys. 108, 013905 (2010).

[21] A. Jaiswal, R. Das, K. Vivekanand, T. Maity, P. M. Abraham, S. Adyanthaya, and P. Poddar, J. Appl. Phys. 107, 013912 (2010).
[22] S. Lei, L. Liu, C. Wang, C. Wang, D. Guo, S. Zeng, B. Cheng, Y. Xiao, and L. Zhou, J. Mater. Chem. A 1, 11982 (2013).

[23] A. McDannald, L. Kuna, and M. Jain, J. Appl. Phys. 114, 113904 (2013).

[24] P. Gupta and P. Poddar, Inorg. Chem. 54, 9509 (2015).

[25] P. Gupta, R. Bhargava, and P. Poddar, J. Phys. D 48, 025004 (2015).

[26] M. Taheri, F. S. Razavi, Z. Yamani, R. Flacau, P. G. Reuvekamp, A. Schulz, and R. K. Kremer, Phys. Rev. B 93, 104414 (2016).

[27] B. Dalal, B. Sarkar, V. D. Ashok, and S. K. De, J. Phys.: Condens. Matter 28, 426001 (2016).

[28] M. Tripathi, R. J. Choudhary, D. M. Phase, T. Chatterji, and H. E. Fischer, Phys. Rev. B 96, 174421 (2017).

[29] B. Dalal, B. Sarkar, V. D. Ashok, and S. K. De, J. Alloys Compd. 739, 418 (2018).

[30] R. D. Johnson, N. Terada, and P. G. Radaelli, Phys. Rev. Lett. 108, 219701 (2012).

[31] J. R. Sahu, C. R. Serrao, N. Ray, U. V. Waghmare, and C. N. R. Rao, J. Mater. Chem. 17, 42 (2007).

[32] A. K. Azad, A. Mellergard, S. G. Eriksson, S. A. Ivanov, S. M. Yunus, F. Lindberg, G. Svensson, and R. Mathieu, Mater. Res. Bull. 40, 1633 (2005).

[33] J. Mao, Y. Sui, X. Zhang, Y. Su, X. Wang, Z. Liu, Y. Wang, R. Zhu, Y. Wang, W. Liu, and J. Tang, Appl. Phys. Lett. 98, 192510 (2011).

[34] J. Mao, Y. Sui, X. Zhang, X. Wang, Y. Su, Z. Liu, Y. Wang, R. Zhu, Y. Wang, W. Liu, and X. Liu, Solid State Commun. 151, 1982 (2011).

[35] N. Dasari, P. Mandal, A. Sundaresan, and N. S. Vidhyadhiraja, Europhys. Lett. 99, 17008 (2012).

[36] T. Bora, P. Saravanan, and S. Ravi, J. Supercond. Nov. Magn. 26, 1645 (2013).

[37] T. Bora and S. Ravi, J. Magn. Magn. Mater. 386, 85 (2015).

[38] F. Pomiro, R. D. Sánchez, G. Cuello, A. Maignan, C. Martin, and R. E. Carbonio, Phys. Rev. B 94, 134402 (2016).

[39] O. V. Billoni, F. Pomiro, S. A. Cannas, C. Martin, A. Maignan, and R. E. Carbonio, J. Phys.: Condens. Matter 28, 476003 (2016). 
[40] M. K. Sharma, K. Singh, and K. Mukherjee, J. Magn. Magn. Mater. 414, 116 (2016).

[41] M. P. Sharannia, S. De, R. Singh, A. Das, R. Nirmala, and P. N. Santhosh, J. Magn. Magn. Mater. 430, 109 (2017).

[42] L. R. Shi, C. X. Wei, Z. Wang, L. Ju, T. S. Xu, T. X. Li, X. W. Yan, and Z. C. Xia, J. Magn. Magn. Mater. 433, 104 (2017).

[43] J. P. Bolletta, F. Pomiro, R. D. Sánchez, V. Pomjakushin, G. Aurelio, A. Maignan, C. Martin, and R. E. Carbonio, Phys. Rev. B 98, 134417 (2018).

[44] J. Lohr, F. Pomiro, V. Pomjakushin, J. A. Alonso, R. E. Carbonio, and R. D. Sánchez, Phys. Rev. B 98, 134405 (2018).

[45] I. Fita, V. Markovich, A. S. Moskvin, A. Wisniewski, R. Puzniak, P. Iwanowski, C. Martin, A. Maignan, R. E. Carbonio, M. U. Gutowska, A. Szewczyk, and G. Gorodetsky, Phys. Rev. B 97, 104416 (2018).

[46] Y. Hosaka, N. Ichikawa, T. Saito, J. P. Attfield, and Y. Shimakawa, Phys. Rev. B 94, 104429 (2016).

[47] A. P. Hammersley, S. O. Svensson, A. Thompson, H. Graafsma, A. Kvick, and J. P. Moy, Rev. Sci. Instrum. 66, 2729 (1995).

[48] H. M. Rietveld, J. Appl. Crystallogr. 2, 65 (1969).

[49] M. Ferrari and L. Lutterotti, J. Appl. Phys. 76, 7246 (1994).

[50] M. W. Lufaso and P. M. Woodward, Acta Crystallogr. B 57, 725 (2001).

[51] V. Siruguri, P. D. Babu, M. Gupta, A. V. Pimpale, and P. S. Goyal, Pramana 71, 1197 (2008).

[52] See Supplemental Material at http://link.aps.org/supplemental/ 10.1103/PhysRevB.101.144418 for the Rietveld fitted roomtemperature SXRD patterns of $x=0.1-0.4$, and 0.6-0.8 samples, Rietveld refined crystallographic parameters, temperature dependent magnetization of $x=0$ and 0.5 sample at various magnetic fields, temperature dependence of structural parameters obtained from the refinement of the NPD patterns at various temperatures for $x=0$ and 0.4 samples, and the expanded view of ZFC and FC $M-H$ loops around the origin at $175 \mathrm{~K}$ for the $x=0.4$ sample.

[53] A. Banerjee, J. Sannigrahi, S. Giri, and S. Majumdar, Phys. Rev. B 98, 104414 (2018).

[54] T. Yamaguchi, J. Phys. Chem. Solids 35, 479 (1974).
[55] P. Mandal, V. S. Bhadram, Y. Sundarayya, C. Narayana, A. Sundaresan, and C. N. R. Rao, Phys. Rev. Lett. 107, 137202 (2011).

[56] K. Zhang, K. Xu, X. Liu, Z. Zhang, Z. Jin, X. Lin, B. Li, S. Cao, and G. Ma, Sci. Rep. 6, 23648 (2016).

[57] I. Dzyaloshinskii, J. Phys. Chem. Solids 4, 241 (1958).

[58] T. Moriya, Phys. Rev. 120, 91 (1960).

[59] M. L. Néel, Ann. Phys. (Paris) 12, 137 (1948).

[60] E. Winkler, S. BlancoCanosa, F. Rivadulla, M. A. LópezQuintela, J. Rivas, A. Caneiro, M. T. Causa, and M. Tovar, Phys. Rev. B 80, 104418 (2009).

[61] R. Nepal, Q. Zhang, S. Dai, W. Tian, S. E. Nagler, and R. Jin, Phys. Rev. B 97, 024410 (2018).

[62] G. T. Lin, Y. Q. Wang, X. Luo, J. Ma, H. L. Zhuang, D. Qian, L. H. Yin, F. C. Chen, J. Yan, R. R. Zhang, S. L. Zhang, W. Tong, W. H. Song, P. Tong, X. B. Zhu, and Y. P. Sun, Phys. Rev. B 97, 064405 (2018).

[63] N. Shamir, H. Shaked, and S. Shtrikman, Phys. Rev. B 24, 6642 (1981).

[64] A. S. Sukhanov, S. Singh, L. Caron, Th. Hansen, A. Hoser, V. Kumar, H. Borrmann, A. Fitch, P. Devi, K. Manna, C. Felser, and D. S. Inosov, Phys. Rev. B 97, 214402 (2018).

[65] A. M. Vibhakar, D. D. Khalyavin, P. Manuel, L. Zhang, K. Yamaura, P. G. Radaelli, A. A. Belik, and R. D. Johnson, Phys. Rev. B 99, 104424 (2019).

[66] P. J. von Ranke, N. A. de Oliveira, C. Mello, D. C. Garcia, V. A. de Souza, and A. M. G. Carvalho, Phys. Rev. B 74, 054425 (2006).

[67] L. Caron, Z. Q. Ou, T. T. Nguyen, D. T. Cam Thanh, O. Tegus, and E. Brück, J. Magn. Magn. Mater. 321, 3559 (2009).

[68] P. J. von Ranke, T. S. T. Alvarenga, B. P. Alho, E. P. Nóbrega, P. O. Ribeiro, A. M. G. Carvalho, V. S. R. de Sousa, A. Caldas, and N. A. de Oliveira, J. Appl. Phys. 111, 113916 (2012).

[69] M. V. deSouza, J. A. daSilva, and L. S. Silva, J. Magn. Magn. Mater. 421, 184 (2017).

[70] L. E. Cross, Ferroelectrics 76, 241 (1987).

[71] Md. G. Masud, A. Ghosh, J. Sannigrahi, and B. K. Chaudhuri, J. Phys.: Condens. Matter 24, 295902 (2012).

[72] Y. Q. Lin and X. M. Chen, J. Am. Ceram. Soc. 94, 782 (2011). 